\title{
Implementation of simultaneous quantitative phase with Raman imaging
}

Nicolas Pavillon ${ }^{1 *}$ and Nicholas I Smith ${ }^{1,2^{*}}$

\author{
*Correspondence: \\ nicolas.pavillon@alumni.epfl.ch; \\ nsmith@ap.eng.osaka-u.ac.jp \\ ${ }^{1}$ Biophotonics Laboratory, \\ Immunology Frontier Research \\ Center (IFReC), Osaka University, \\ Yamadaoka 3-1, 562-0871 Suita, \\ Osaka, Japan \\ 2 PRESTO, Japan Science and \\ Technology Agency (JST), \\ Chiyodaku 102-0075, Tokyo, Japan
}

\begin{abstract}
We present a technical overview of a multimodal system combining Raman microspectroscopy and quantitative phase microscopy (QPM), which allows two independent and simultaneous measurements of both the local molecular content and dynamic sample morphology. We present in detail the setup implementation and measurement procedure, and show how different features of QPM can be used to ensure optimal Raman measurement conditions and matched fields of view, through off-line calibration procedures such as digital propagation of the measured complex field and analysis of the system's optical aberrations which can then be employed for numerical compensation and calibration. We present measurements on live cells, where images based both on the quantitative phase signal and on the Raman molecular contrast can simultaneously be retrieved and compared. The dynamic measurements obtained from QPM also enable the monitoring of the cell morphology during the laser scanning of the Raman measurement, making it possible to identify the movements which may occur during the measurement.
\end{abstract}

PACS codes: $87.64 .-\mathrm{t} ;$ 87.64.kp; 42.40.Kw

Keywords: Raman spectroscopy; Digital holography; Microscopy; Multimodal imaging; Aberration compensation; Live cell imaging; Label-free

\section{望 Springer}

\section{Introduction}

Raman spectroscopy is a valuable tool for non-invasive optical analysis, based on the measurement of the inelastic scattering of a laser excitation, where resonant chemical bonds in the sample possess specific energy shifts corresponding to their various vibrational modes. In the context of biological samples, the Raman spectral information has been used to classify between different cell lines [1,2], or detect cancerous cells or tissue [3,4], among many other applications.

As the excitation light is situated in the visible wavelength range, the technique possesses a spatial resolution which makes spectral imaging possible by coupling the spectral detection with laser-scanning systems. Due to recent advances in the development of low-noise two-dimensional detectors, the number of applications based on Raman microscopy has significantly grown in the past years, to observe, for instance, the distribution of molecules or organelles within cells, such as mitochondria [5], cytochrome c [6] or fatty acids [7].

(c) 2015 Pavillon and Smith; licensee Springer on behalf of EPJ. This is an Open Access article distributed under the terms of the Creative Commons Attribution License (http://creativecommons.org/licenses/by/4.0), which permits unrestricted use, distribution, and reproduction in any medium, provided the original work is properly credited. 
However, as the Raman signal is usually weak, in particular for biological samples, imaging measurements with extended areas still require rather high excitation power and take a significant amount of time for the complete measurement, which is typically in the range of minutes to tens of minutes, which makes the observation of dynamic phenomena with Raman microscopy very challenging.

To enable the monitoring of dynamic phenomena or the observation of wide areas, Raman microspectroscopy has often been used sequentially with other imaging methods, such as auto-fluorescence [8], optical coherence tomography [9], or fluorescence [10], to cite some of the numerous applications. We present here a measurement approach where the Raman channel is coupled with another imaging modality which can be recorded simultaneously and provides a fast acquisition rate, enabling the dynamic monitoring of the samples during the slower Raman measurement. This second modality is based on quantitative phase microscopy (QPM), implemented as a digital holographic microscope (DHM), a wide-field imaging technique which enables the measurement of the quantitative phase shifts induced by the sample [11]. DHM presents the advantage of retaining the label-free feature of Raman microscopy, while enabling simultaneous measurements based on spectral filtering. While combining other modalities with the Raman signal is not trivial since the Raman scattering is a weak, unpolarised response with a large bandwidth, the combination with DHM can be readily performed by choosing a low power laser for holography at a wavelength outside of the Raman emission range [12]. Additionally, choosing a DHM mode wavelength longer than the Raman region of interest additionally ensures that any Stokes-shifted fluorescence generated remains outside the Raman region of interest.

Quantitative phase signals have recently been used to derive biological indicators for live cell observation, such as the measurement of dry mass [13], cell differentiation based on morphology [14], or early indicators of cell death [15], to cite some possible applications. The multimodal measurements based on Raman microscopy and QPM lead to complimentary information, where Raman can provide information on the molecular content, while QPM enables the dynamic monitoring of the sample morphology. Furthermore, additional correlation between the two signals can be derived, as both are based on different physical interactions with the sample [16].

We present a methodology and results for this type of approach, first by showing multimodal measurements on latex beads, to characterise the imaging in both channels. We then describe one of the possible procedures that can calibrate such a multimodal system for precise co-localisation in both modes. Measurements on live cells are then presented, which show the possibility of dynamically monitoring the cell morphology by phase during the Raman measurements. Further details on the setup implementation and the image reconstruction procedures are provided in the Methods section.

\section{Results}

The combined measurement procedure is presented first through measurements on latex beads, which can be employed to visualise the field of view in both the Raman and QPM channels. This allows us to quantify the degree of spatial mismatch between the two modes, which can then be used as a basis to compensate or calibrate the overall system. In a second part, measurements on live fibroblasts cells are presented to illustrate 
the combined dynamic and spectral information which can be retrieved through these multimodal measurements.

\section{Image calibration}

As the Raman and QPM channels are measured with different detectors, it is necessary to adjust the fields of view in order to obtain comparable images. This is particularly important as the two cameras will usually have significantly different chip sizes. The low-noise cameras used for Raman detection usually have rather large pixel sizes (in the order of $10-15 \mu \mathrm{m}$ ), while preferred detectors for off-axis DHM have smaller pixel sizes (typically around $5 \mu \mathrm{m}$ ), to ensure a high spatial sampling of the interference fringes. In our implementation, to partially compensate for these differences, the relay optics in the DHM system as described in Methods are designed to provide a demagnification of $1.5-2 \times$ to first give an approximate match between the fields of view on the two detectors. It is then possible to register the two images in order to compensate for the remaining differences. Disregarding, for the moment, any complex distortions or aberrations, the main differences between the two modes should be accounted for by some amounts for translation (detector position mismatch), rotation (detector orientation mismatch) and magnification (field of view mismatch). These amounts can then be determined by finding the maximum value of the two-dimensional cross-correlation between the Raman image $I_{R}$ and the adjusted DHM image $I_{H}$, which corresponds mathematically to maximising the problem

$$
\beta\left(\boldsymbol{\alpha}, \boldsymbol{x}_{\mathbf{0}}, \theta\right)=\max \{C(x, y)\}, \quad C(x, y)=R(\theta) \cdot I_{H}\left(\alpha_{x} x, \alpha_{y} y\right) \star I_{R}(x, y),
$$

where $\star$ is the correlation operator, $\boldsymbol{\alpha}$ is a spatial scaling factor, $R(\theta)$ is a rotation operator of an angle $\theta$, and $x_{0}$ is the position of the maximum value of $C(x, y)$, corresponding to a translation.

This procedure is illustrated in Figure 1, where $0.8 \mu$ m diameter polystyrene beads fixed onto a quartz substrate and then immersed in water have been measured with both channels, providing precise locations to calibrate the position of the two detectors. The beads distribution can been seen in the Raman channel (cf. Figure 1a), along with the spectrum of the beads (cf. Figure 1b), where the image has been extracted by highlighting the $1003 \mathrm{~cm}^{-1}$ band. The spectrum also contains a significant contribution coming from the quartz substrate, since the beads are in contact with it. The corresponding hologram is shown in Figure 1c, where the diffraction patterns of the beads can be identified on top of the interference fringes. The amplitude of the reconstructed wave front (propagated into focus) is shown in Figure 1d, where beads can be seen as black dots, as they strongly diffract the incoming light, with the approximate Raman field of view highlighted by the dashed rectangle.

The result of the registration procedure as described in Eq. (1) is shown in Figure 1e, where the two channels are merged together with the Raman channel in red and the inverted DHM amplitude in green. The linear transformations lead to a registration accuracy of \pm 5 pixels, where the location differences are oriented in different directions across the field of view. This shows that the remaining inaccuracies are induced by distortions such as aberrations in the two detection arms.

One approach to obtain a higher registration accuracy would be to correct the excitation laser locations during acquisition, by using maps as shown in Figure 1e to adjust 


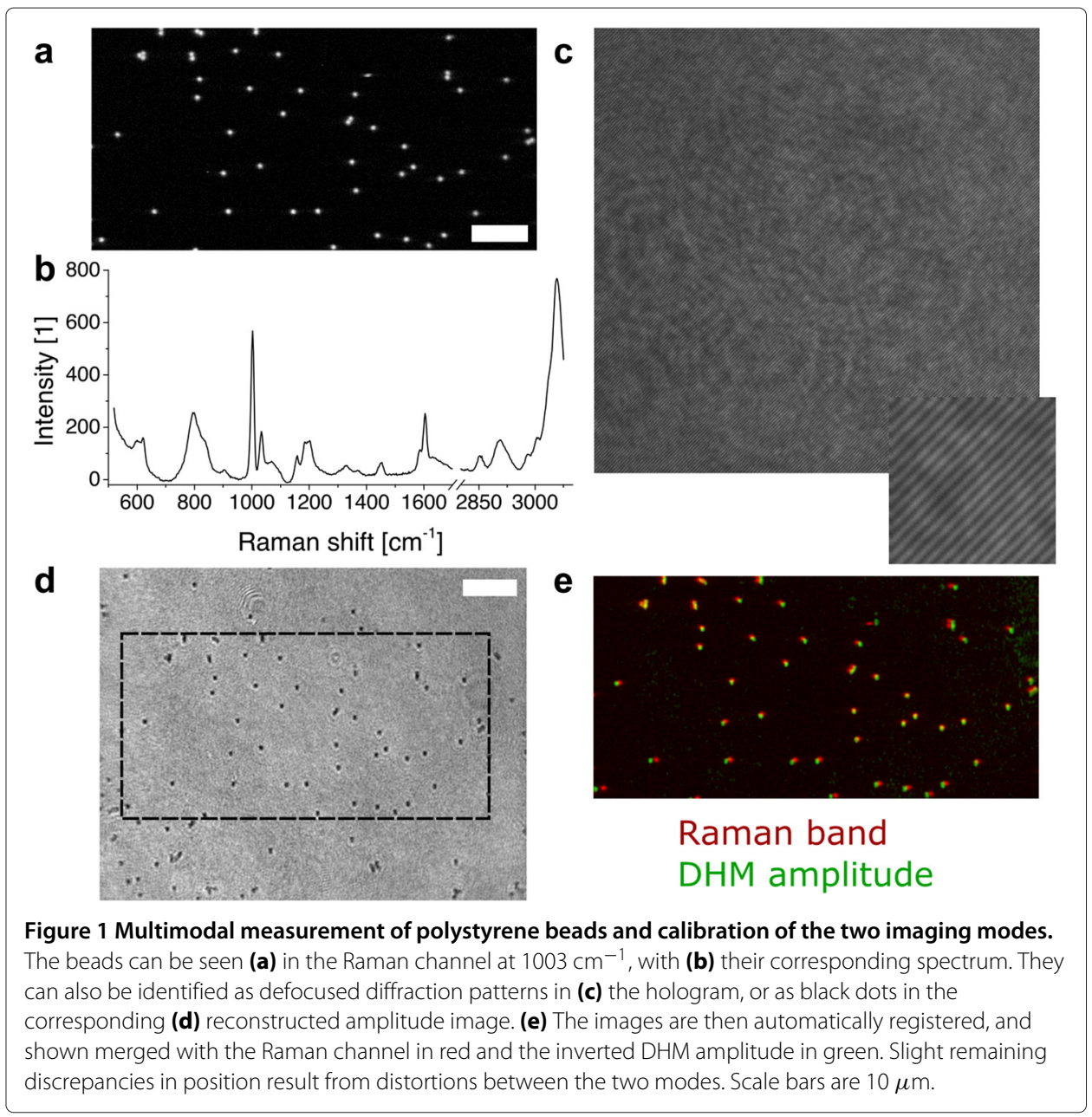

the driving voltages of the galvano-mirrors, and generate an equally spaced spatial sampling in the object space. However, this approach is not suited for a system based on a slit scanning configuration, as several locations are detected in parallel on the equally-spaced pixels of a 2D detector.

Another approach is to numerically adjust the DHM reconstruction to match the Raman image resulting from the possibly aberrated optical system. This can be achieved by employing phase masks of higher order, denoted as numerical parametric lenses (NPL) [17] to simulate the existing aberrations in the system, and obtain a corrected phase image. This approach is usually employed to compensate the existing aberrations in order to retrieve a flat phase in the field of view, as shown for example in the live cells measurements of the next section, but can also be employed to induce specific aberrations. In this case, the same type of optimisation as described in Eq. (1) can be employed, where the adjusted DHM image $I_{H}$ is now extracted from a modified wave front $\Psi^{\prime}(x, y)$, generated from the original wave front $\Psi(x, y)$ multiplied with a phase mask $\varphi_{\mathrm{NPL}}$, defined as

$$
\begin{aligned}
\varphi_{\mathrm{NPL}}(x, y) & =\sum_{i=0}^{P} \sum_{j=0}^{P}\left(c_{x, i} x^{i}+c_{y, j} y^{j}\right), \\
\Psi^{\prime}(x, y) & =\Psi(x, y) \cdot \exp \left[-\mathrm{i} \varphi_{\mathrm{NPL}}(x, y)\right],
\end{aligned}
$$


where $\varphi_{\mathrm{NPL}}$ is generated from a polynomial expansion of degree $P$ in both $x$ and $y$ directions, and $c_{x, i}, c_{y, i}$ are the coefficients to be adjusted to improve the overlay of beads in the two images. The amplitude image can then be extracted from the modified wave front $\Psi^{\prime}$ propagated info focus (see Eq. (4)).

The images shown in Figure 1e were treated based on the approach described in Eq. (2), with an NPL generated from a polynomial of degree $P=12$. The resulting registration is shown in Figure 2a, where the beads are co-localised in the upper-right part of the image compared to the original shifts, while there is a remaining mismatch in the lower-left part of the image, which now appears to be constant. The NPL leading to this registration is shown in Figure 2b. It contains phase differences, as visualised by the wrapping of the phase, in the range of 3 wavelengths, which could be considered rather high in terms of aberrations. However, a significant part of the profile is originating from a tilt, which may be induced by a remaining mismatch in the detectors position. Removing this linear term in the NPL mask shows that the aberration level in the region of interest corresponds to approximately one wavelength, as shown in Figure 2c.

\section{Live cells multimodal measurements}

The multimodal measurements are here illustrated by measuring live mouse embryonic fibroblasts cells (MEF) [18], cultured in Dulbecco's modified Eagle medium, and plated on quartz-bottom dishes one day prior experiment. Before observation, the culture medium was replaced with a phosphate buffer saline solution supplemented with glucose.

A typical measurement is shown in Figure 3, where the MEF cells have been observed in phase (see Figure 3a-b) during the Raman measurement with an acquisition every $1.5 \mathrm{~s}$, and a Raman exposure time of $3 \mathrm{~s}$ per line, leading to a measurement time of 10 minutes. It is possible to identify the cell morphology changes between the beginning (Figure 3a) and the end of the measurement (Figure $3 \mathrm{~b}$ ), with a slight change in shape, reorganisation of small lipid structure, or movements of nucleoli. The corresponding Raman image is shown in Figure 3c, where two bands (red: 2865, green: $2973 \mathrm{~cm}^{-1}$ ) have been highlighted, respectively corresponding to $\mathrm{CH}_{2}$ and $\mathrm{CH}$ stretching, which can be attributed to lipids and global content of the cell. Due to the molecular contrast, the Raman image shows significantly more information than the phase ones. On the other hand, the full evolution of the cell morphology during the laser excitation scan is shown in the QPM channel (cf. Additional file 1), where significant movements of organelles, and in particular small
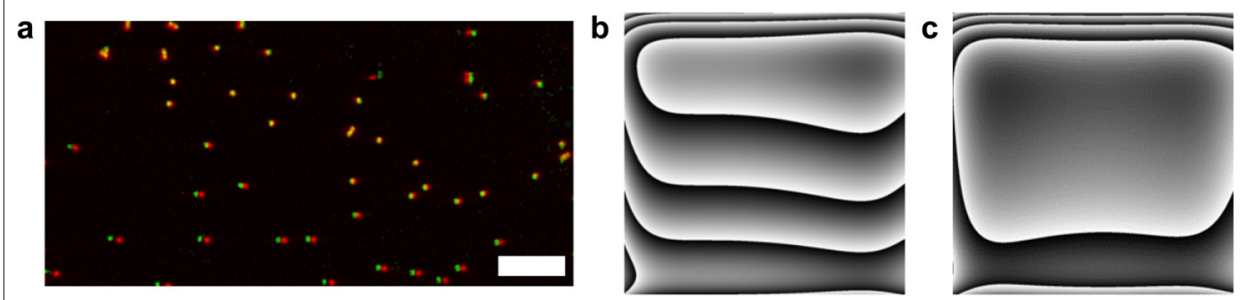

Figure 2 Image registration through aberration compensation. (a) Registered latex beads images, merged with the Raman channel in red and the inverted DHM amplitude in green, where a NPL has been used to improve the overlay of beads compared to Figure 1e. (b) Wrapped NPL employed to generate (a). (c) Wrapped NPL mask, with linear (tilt) term removed, which represents the aberration mismatch between both detection arms. 


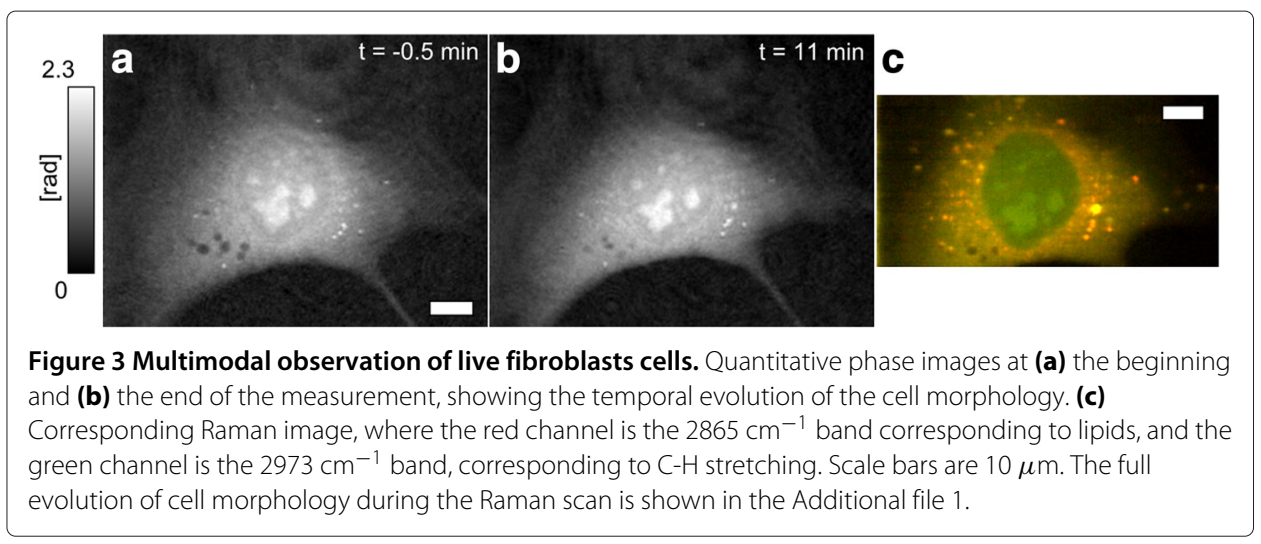

lipids bodies, can be identified. It is also possible to see that the whole cell is not clearly visible in the Raman channel, where the external part does not provide much signal. This can be attributed to the lower quantity of biomolecules in the very thin parts of the cell, but also in part to the optical sectioning inherent to Raman, which was located in the centre of the cell, and not on the substrate.

In order to emphasise the changes which can occur during the laser excitation scan, a second set of measurements was performed with approximately two times the power density employed in the other measurements, to $4.34 \mathrm{~mW} / \mu \mathrm{m}^{2}$. The result is shown in the Additional file 2, where it is possible to identify the large morphology changes following the laser scan at higher power, which induces errors in the Raman image, such as the sharp change in the left border of the cell, which results from rapid cell shrinkage. In this case, phase images were recorded every $1.5 \mathrm{~s}$, which is sufficient to follow the morphology changes of the cell, but the acquisition capability is generally limited by the recording speed of the camera. This then typically allows video-rate acquisition, so that faster events could also be observed, while the Raman imaging channel typically requires exposures in the order of seconds per line for biological samples, corresponding to an image acquisition on the order of minutes.

The multimodal approach presented above enables the measurement of two sets of information, with the Raman channel providing spectral information related to the molecular content of the sample, and the phase measurement being a quantitative indicator of the sample dynamic morphology. As shown with the measurements above, the simultaneous acquisition of the two can be employed for samples where the imaging speed of the Raman channel may not be fast enough to follow dynamic changes which can occur during the acquisition.

These combined measurements could also prove useful in other fields such as material science, where speed is of less concern but where both techniques can provide specific features. Raman spectroscopy has been extensively used in this field to characterize material composition and structure [19], and DHM can be employed in conjunction to provide full-field profilometry with a resolution in the order of $10 \mathrm{~nm}$ and below [20,21]. Furthermore, the two measurements are physically complimentary, with QPM and Raman being related respectively to the elastic and inelastic scattering of the excitation light in the sample, enabling the comparison of these two sets of data to derive additional information [16]. 


\section{Methods}

As the presented measurement approach is based on multimodal measurements, the different channels, namely Raman imaging and DHM, are first presented independently. The combination of the two data sets is then discussed in a following section.

\section{Raman setup}

The Raman setup is an imaging system based on an epi-excitation configuration as shown in Figure 4a. Our implementation is shown in Figure 4b, with the beam paths highlighted. The excitation is provided by a continuous wave laser at $532 \mathrm{~nm}$, which is injected into a commercial microscope base by focusing the laser onto the image plane of an output port of the microscope. The excitation is then tightly focused by a microscope objective $(60 \times$, NA 1.27 , water), which also collects the back-scattered light. The red-shifted emitted light is separated from the excitation with a short-pass dichroic mirror, and imaged back onto the slit of a spectrometer through relay optics. A Czerny-Turner spectrometer (focal length $500 \mathrm{~mm}$ ) then disperses the light with a reflective grating and images back the spectral line onto a low noise scientific CMOS detector. To enable imaging, two scanning mirrors are employed in the path of the excitation laser, and placed at conjugate planes corresponding to Fourier planes of the focal spot. The setup is arranged in a slitscanning configuration, to enable parallel detection of all spectra on a line of the sample onto the two-dimensional detector [22]. In this case, the first mirror (horizontal scanning) is placed in the descanned region of the setup, so that a displaced spot stays focused on the slit of the spectrometer, while the second mirror (vertical scanning, parallel to slit) is placed before the dichroic mirror, so that the scanning follows the slit direction. The experiments were performed with a low-frequency grating $(300 \mathrm{lp} / \mathrm{mm})$ to acquire both the fingerprint and $\mathrm{CH}$ stretching region, with a slit width of around $60 \mu \mathrm{m}$, leading to a spectral resolution of approximately $12 \mathrm{~cm}^{-1}$.

The laser delivers an approximate power of $100 \mathrm{~mW}$ on the sample, which corresponds to a power density of $480 \mathrm{~mW} / \mu \mathrm{m}^{2}$ at the focal spot. To generate the line excitation, the spot is then rapidly scanned at $100 \mathrm{~Hz}$ by the vertical mirror along the measurement zone during the excitation duration in the seconds range, leading to an average power density of $2.47 \mathrm{~mW} / \mu \mathrm{m}^{2}$.

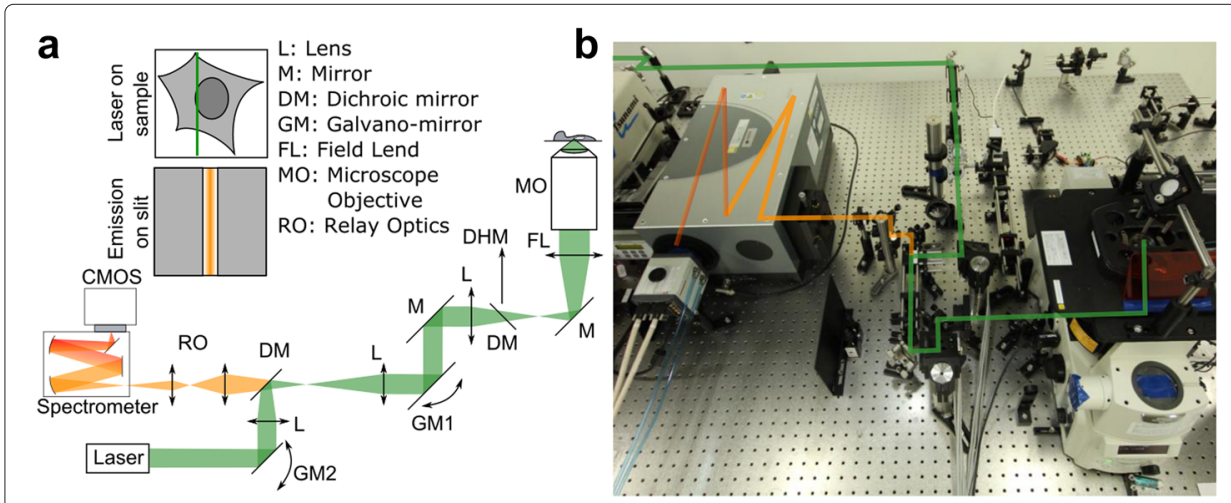

Figure 4 Schematic and implementation of the Raman setup. (a) Schematic of Raman setup in the slit-scanning configuration. (b) Practical implementation of the optical setup. 


\section{DHM setup}

The DHM setup is a wide-field microscope, where the hologram is recorded based on a Mach-Zehnder interferometer, as schematically shown in Figure 5a, with the practical implementation shown in Figure 5b, which also shows the beam paths. The light emitted by a laser diode is spatially filtered through a pinhole to obtain a homogeneous wave front with high spatial coherence, which is then split into an object and reference arm. The object beam is loosely focused onto the sample by a condenser lens to illuminate the field of view, and the diffracted light is collected by the microscope objective. The image plane of the microscope base is conjugated near the detector by relay optics, after which the object and reference beams are recombined by a beamsplitter. The interference signal is detected in the Fresnel regime by a CCD camera. The diode delivers approximately $400 \mu \mathrm{W}$ of power evenly distributed on the sample, with the hologram being recorded during an exposure time in the milliseconds range. The recorded hologram contains both pure intensity terms (zero-order) as well as the coherent orders (+1/-1 diffraction terms) as defined by

$$
I(x, y)=|o+r|^{2}=|o|^{2}+|r|^{2}+o^{*} r+o r^{*},
$$

where $o, r$ are respectively the object and reference beams, and ()$^{*}$ is the complex conjugate operator. The interferometer is tuned to be in an off-axis configuration, where the reference beam propagation direction is slightly tilted from the optical axis, in order to obtain high frequency interference fringes, and consequently spatially modulate the coherent terms of the hologram. This enables retrieval of the measured complex field, corresponding to the coherent term $o r^{*}$, from the intensity values of the hologram through Fourier filtering, where the coherent term is spectrally separated from the zero-order and conjugate terms [23]. The retrieved complex field can then be demodulated and compensated for aberrations by employing a measurement of the system response [24].

The use of an interferometer configuration implies that the measurement may be more sensitive to vibrations compared to other types of implementations such as common-path systems [25]. However, in the implementation chosen here, the short exposure time and the one-shot acquisition provided by the off-axis configuration prevent vibrations below
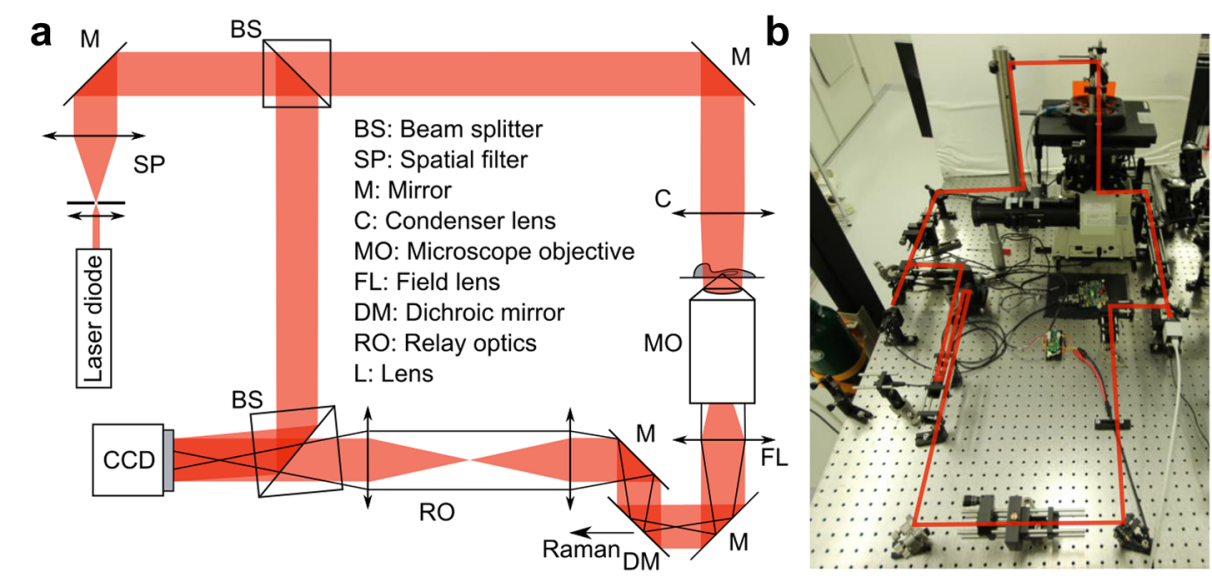

Figure 5 Schematic and implementation of the DHM setup. (a) Schematic of the digital holographic microscope, along with (b) its practical implementation. 
$\mathrm{kHz}$ frequency from influencing the measurement. Furthermore, this approach provides more flexibility for the implementation of multimodal systems as there is no need to generate a reference wave from the imaging part of the system, as is required in selfreferenced common-path configurations. It also makes it possible to independently tune and adjust the object and reference waves to optimize image quality.

\section{Imaging modes combination}

The systems presented in the previous subsections can be combined to provide simultaneous multimodal measurements by separating the signals spectrally. The wavelength employed for DHM measurements can be freely chosen and can therefore be selected to be out of the range of the Raman emission, and was selected here in the near-infrared region where absorption is minimal $(\lambda \simeq 780 \mathrm{~nm}$ ), which corresponds to a Raman shift of $\sim 6000 \mathrm{~cm}^{-1}$ for the excitation wavelength of $532 \mathrm{~nm}$, well outside the Raman range of interest.

In case the spectral ranges for the two channels are fundamentally separate, the two measurements are fully independent, as even stray light or imperfections in spectral separation optics do not influence the signals. On one hand, DHM is wide-field, so that remaining light impinging on the spectrometer is not focused, and is thus strongly filtered by the slit. On the other hand, light from the Raman excitation laser which would be detected by the DHM detector in case of imperfections in the dichroic mirror would not interfere with the reference arm light, and would be filtered out during the Fourier reconstruction process.

As the two channels are measured with different detectors, it is necessary to ensure that both images are in focus. This is particularly important for Raman imaging, where the measured signal intensity is highly dependent on the tightly focused excitation power. It is possible to employ here the digital propagation feature of DHM, which makes it possible to numerically focus the image based on the information from the measured wave front encoded in the hologram. The focus can therefore be experimentally adjusted during measurement to record an optimal Raman signal, while the quantitative phase can be focused off-line by employing numerical algorithms such as the angular spectrum, defined as [26]

$$
\begin{gathered}
A\left(\omega_{x}, \omega_{y}, d\right)=A\left(\omega_{x}, \omega_{y}, 0\right) \cdot \exp \left(\mathrm{i} \frac{2 \pi}{\lambda} d \sqrt{1-\left(\lambda \omega_{x}\right)^{2}-\left(\lambda \omega_{y}\right)^{2}}\right), \\
A\left(\omega_{x}, \omega_{y}, z\right)=\mathscr{F}\{\Psi(x, y, z)\},
\end{gathered}
$$

where $d$ is the propagation distance, $\mathscr{F}\{\cdot\}$ denotes the Fourier transform, $\left(\omega_{x}, \omega_{y}\right)$ are the spatial frequencies, and $\Psi(x, y, z)$ is the spatial complex field defined at the $z$ position.

\section{Conclusion}

We presented the implementation of a multimodal microscope which combines Raman imaging, based on a parallel detection scheme through a line scanning configuration, and quantitative phase microscopy (QPM) based on off-axis digital holographic microscopy (DHM). The two modes can be measured independently and simultaneously through spectral separation of the two wavelength regimes, where the laser line employed in DHM can be freely chosen outside the Raman emission range. This approach makes it possible to combine the specific information from the Raman channel, based on the detection 
of the intracellular molecular contrast, and the quantitative phase signal, which provides information about the sample morphology and can be measured at video-rate for dynamic monitoring.

As QPM is also based on a label-free contrast, samples can be measured without requiring specific preparation before observation, thus retaining one of the main features of Raman spectroscopy. Furthermore, QPM also employs a reconstruction procedure where several steps are performed off-line after measurement, which can be used in the context of multimodal acquisition to simplify or enhance the measurement procedure. As the measured complex field can be numerically propagated into focus, the actual measurements can be optimised for the Raman channel, whose signal quality is highly sensitive to precise focus of the excitation light within the sample, while allowing post-processing calibration of focus in the QPM mode, thereby ensuring sharp images in both channels without complicated setup adjustments.

As well as providing significant morphological information and temporal dynamics of the sample, the quantitative information retrieved by QPM can also be employed to characterise the setup itself, by determining the amount of aberration present in the system. While aberrations are usually compensated in DHM through a calibration step in order to obtain a flat phase profile, this approach can also be used to estimate the aberrations present in the Raman channel, and pre-compensate for the possible mismatches induced by the aberration differences within the different arms of the microscope system.

Measurements on live cells show that the acquisition rate of the Raman channel, inherently limited by the weak Raman signal and photo-toxicity, may not be fast enough to follow some rapid changes which can occur during the measurement.

The presented approach extends the range of standard Raman measurements, where it becomes possible to extract both spatial and molecular information from spectroscopy, but also dynamic morphological information from the quantitative phase.

\section{Additional files}

Additional file 1: Time course of multimodal measurements of MEF cells from Figure 3. The phase images (left) recorded every $1.5 \mathrm{~s}$ show the evolution of the cell morphology and organelle movements during the Raman measurement (right), which is performed through line scanning. The total time of the measurement is $11.5 \mathrm{~min}$, and the field of view of the DHM image is $70 \times 93 \mu \mathrm{m}$.

Additional file 2: Time course of multimodal measurements of MEF cells. The phase images (left) recorded every $1.5 \mathrm{~s}$ show the evolution of the cell morphology during the Raman measurement (right), which is performed through line scanning. It is possible to identify a dramatic cell shrinkage under the high power excitation. The total time of the measurement is $27.5 \mathrm{~min}$, and the field of view of the DHM image is $90 \times 83 \mu \mathrm{m}$.

Competing interests

The authors declare that they have no competing interests.

Authors' contributions

NP and NIS designed the experiment and drafted the manuscript. NP carried out measurements and performed data analysis. Both authors read and approved the final manuscript.

\section{Acknowledgements}

The authors would like to thank Dr. T. Saitoh and Dr. S. Akira (Osaka University) who donated the spontaneously immortalized wild-type mouse embryonic fibroblasts.

This work was funded by the Japan Society for the Promotion of Science (JSPS) through the Funding Program for World-Leading Innovative R\&D on Science and Technology (FIRST Program), and by the JSPS World Premier International Research Center Initiative Funding Program, and Japan Science and Technology Agency PRESTO program. 


\section{References}

1. Notingher I, Jell G, Lohbauer U, Salih V, Hench LL. In situ non-invasive spectral discrimination between bone cell phenotypes used in tissue engineering. J Cell Biochem. 2004;92(6):1180-92.

2. Swain RJ, Kemp SJ, Goldstraw P, Tetley TD, Stevens MM. Assessment of cell line models of primary human cells by Raman spectral phenotyping. Biophys J. 2010;98(8):1703-11.

3. Haka AS, Shafer-Peltier KE, Fitzmaurice M, Crowe J, Dasari RR, Feld MS. Diagnosing breast cancer by using Raman spectroscopy. Proc Natl Acad Sci U S A. 2005;102(35):12371-6.

4. Lloyd GR, Orr LE, Christie-Brown J, McCarthy K, Rose S, Thomas M, et al. Discrimination between benign, primary and secondary malignancies in lymph nodes from the head and neck utilising Raman spectroscopy and multivariate analysis. Analyst. 2013;138(14):3900-8.

5. Matthäus C, Chernenko T, Newmark JA, Warner CM, Diem M. Label-free detection of mitochondrial distribution in cells by nonresonant Raman microspectroscopy. Biophys J. 2007;93(2):668-73.

6. Okada M, Smith NI, Palonpon AF, Endo H, Kawata S, Sodeoka M, et al. Label-free Raman observation of cytochrome c dynamics during apoptosis. Proc Natl Acad Sci U S A. 2012;109(1):28-32.

7. Hedegaard M, Krafft C, Ditzel HJ, Johansen LE, Hassing S, Popp J. Discriminating isogenic cancer cells and identifying altered unsaturated fatty acid content as associated with metastasis status, using K-Means clustering and partial least squares-discriminant analysis of Raman maps. Anal Chem. 2010;82(7):2797-802

8. Kong K, Rowlands CJ, Varma S, Perkins W, Leach $1 \mathrm{H}$, Koloydenko AA, et al. Diagnosis of tumors during tissue-conserving surgery with integrated autofluorescence and raman scattering microscopy. Proc Natl Acad Sci U S A. 2013;110(38):15189-94.

9. Patil CA, Kalkman J, Faber DJ, Nyman JS, van Leeuwen TG, Mahadevan-Jansen A. Integrated system for combined Raman spectroscopy-spectral domain optical coherence tomography. J Biomed Opt. 2011;16(1):011007.

10. van Manen H-J, Kraan YM, Roos D, Otto C. Single-cell Raman and fluorescence microscopy reveal the association of lipid bodies with phagosomes in leukocytes. Proc Natl Acad Sci U S A. 2005;102(29):10159-64.

11. Marquet P, Rappaz B, Magistretti PJ, Cuche E, Emery Y, Colomb T, et al. Digital holographic microscopy: a noninvasive contrast imaging technique allowing quantitative visualization of living cells with subwavelength axial accuracy. Opt Lett. 2005;30(5):468-70.

12. Pavillon N, Fujita K, Smith NI. Multimodal label-free microscopy. J Innov Opt Health Sci. 2014;7(5):1330009.

13. Rappaz B, Cano E, Colomb T, Kühn J, Simanis V, Magistretti PJ, et al. Noninvasive characterization of the fission yeast cell cycle by monitoring dry mass with digital holographic microscopy. J Biomed Opt. 2009;14(3):034049.

14. Chalut KJ, Ekpenyong AE, Clegg WL, Melhuish IC, Guck J. Quantifying cellular differentiation by physical phenotype using digital holographic microscopy. Integr Biol (Camb). 2012;4(3):280-4

15. Pavillon N, Kühn J, Moratal C, Jourdain P, Depeursinge C, Magistretti PJ, et al. Early cell death detection with digital holographic microscopy. PLoS ONE. 2012;7(1):30912.

16. Pavillon N, Hobro AJ, Smith NI. Cell optical density and molecular composition revealed by simultaneous multimodal label-free imaging. Biophys J. 2013;105(5):1123-32.

17. Colomb T, Cuche E, Charrière F, Kühn J, Aspert N, Montfort F, et al. Automatic procedure for aberration compensation in digital holographic microscopy and applications to specimen shape compensation. Appl Opt. 2006;45(5):851-63.

18. Saitoh T, Fujita N, Hayashi T, Takahara K, Satoh T, Lee H, et al. Atg9a controls dsDNA-driven dynamic translocation of STING and the innate immune response. Proc Natl Acad Sci U S A. 2009;106(49):20842-6.

19. Chalmers JM, Griffiths PR. Handbook of vibrational spectroscopy, vol. 4: Applications in industry, materials and the physical sciences. New York: John Wiley \& Sons; 2001

20. Coppola G, Ferraro P, lodice M, Nicola SD, Finizio A, Grilli S. A digital holographic microscope for complete characterization of microelectromechanical systems. Meas Sci Technol. 2004;15(3):529-39.

21. Kühn J, Charrière F, Colomb T, Cuche E, Montfort F, Emery Y, et al. Axial sub-nanometer accuracy in digital holographic microscopy. Meas Sci Technol. 2008;19(7):074007.

22. Hamada K, Fujita K, Smith NI, Kobayashi M, Inouye Y, Kawata S. Raman microscopy for dynamic molecular imaging of living cells. J Biomed Opt. 2008;13(4):044027.

23. Cuche $E$, Marquet $P$, Depeursinge $C$. Simultaneous amplitude-contrast and quantitative phase-contrast microscopy by numerical reconstruction of Fresnel off-axis holograms. Appl Opt. 1999;38(34):6994-7001.

24. Colomb T, Kühn J, Charrière F, Depeursinge C, Marquet $P$, Aspert N. Total aberrations compensation in digital holographic microscopy with a reference conjugated hologram. Opt Express. 2006;14(10):4300-6.

25. Popescu G, Ikeda T, Dasari RR, Feld MS. Diffraction phase microscopy for quantifying cell structure and dynamics. Opt Lett. 2006;31(6):775-7.

26. Goodman JW. Introduction to fourier optics, 2nd edn. New York: Mc Graw Hill Companies, Inc.; 1996. 